\title{
Evolution of the Luminosity Density in the Universe: Implications for the Nonzero Cosmological Constant
}

\author{
T. $\operatorname{Totani}^{1}$, Y. Yoshii ${ }^{2,3}, \&$ K. Sato ${ }^{1,3}$
}

\begin{abstract}
We show that evolution of the luminosity density of galaxies in the universe provides a powerful test for the geometry of the universe. Using reasonable galaxy evolution models of population synthesis which reproduce the colors of local galaxies of various morphological types, we have calculated the luminosity density of galaxies as a function of redshift $z$. Comparison of the result with recent measurements by the Canada-France Redshift Survey in three wavebands of $2800 \AA, 4400 \AA$, and $1 \mu \mathrm{m}$ at $z<1$ indicates that the $\Lambda$-dominated flat universe with $\lambda_{0} \sim 0.8$ is favored, and the lower limit on $\lambda_{0}$ yields 0.37 (99\% C.L.) or 0.53 (95\% C.L.) if $\Omega_{0}+\lambda_{0}=1$. The Einstein-de Sitter universe with $\left(\Omega_{0}, \lambda_{0}\right)=(1,0)$ and the low-density open universe with $(0.2,0)$ are however ruled out with 99.86\% C.L. and $98.6 \%$ C.L., respectively. The confidence levels quoted apply unless the standard assumptions on galaxy evolution are drastically violated.

We have also calculated a global star formation rate in the universe to be compared with the observed rate beyond $z \sim 2$. We find from this comparison that spiral galaxies are formed from material accretion over an extended period of a few Gyrs, while elliptical galaxies are formed from initial star burst at $z \gtrsim 5$ supplying enough amount of metals and ionizing photons in the intergalactic medium.
\end{abstract}

Subject headings: galaxies: evolution - galaxies: formation - galaxies: intergalactic medium - cosmology: theory

\footnotetext{
${ }^{1}$ Department of Physics, School of Science, the University of Tokyo, Tokyo 113, Japan

E-mail: totani@utaphp2.phys.s.u-tokyo.ac.jp

${ }^{2}$ Institute of Astronomy, Faculty of Science, the University of Tokyo, 2-21-1 Osawa, Mitaka, Tokyo 181, Japan

${ }^{3}$ Research Center for the Early Universe, School of Science, the University of Tokyo, Tokyo 113, Japan
} 


\section{Introduction}

Recent progress in deep redshift surveys of galaxies reaching $z \sim 1$ gives valuable information on the history of galaxy evolution (Lilly et al. 1995; Ellis et al. 1996). The Canada-France Redshift Survey (CFRS) consisting of $730 I_{A B}$-selected galaxies with $17.5<I_{A B}<22.5$ revealed a marked evolution in their comoving luminosity density in three wavebands of $2800 \AA, 4400 \AA$, and $1 \mu \mathrm{m}$ in the range of $z=0-1$ (Lilly et al. 1996). They argued that their data can be explained by a galaxy evolution model of population synthesis with star formation rate proportional to $\tau^{-2.5}$ where $\tau$ is the elapsed time from the initial turn-on of star formation. In practice, however, their use of a single index -2.5 for all galaxies is not justified because the present-day colors of galaxies are strongly correlated with their morphological type through type-dependent history of star formation (Tinsley 1980), and a single-type analysis is known to produce a bias in interpreting the data when applied to a sample with a realistic mixture of morphological types (Yoshii \& Takahara 1989). One therefore has to construct type-dependent evolution models of galaxies and sum up with relative proportion of their types. If the global history of star formation for the composite of all types of galaxies is determined in this way, cosmological models can then be tested against the observations of the luminosity density evolution in the universe.

In this Letter, we calculate an evolution of the luminosity density using the galaxy evolution models of population synthesis developed by Arimoto \& Yoshii (1987, hereafter AY) and Arimoto, Yoshii, \& Takahara (1992, hereafter AYT) where the effect of chemical evolution is appropriately taken into account. Since the integrated color of stars in a galaxy is very sensitive to an assumed history of star formation, the present-day spectral energy distribution (SED) in the ultraviolet through near-infrared region for each type of galaxies can only be reproduced by almost identical luminosity evolution as a function of look-back time until the epoch corresponding to an average age of stars in a galaxy (AY, AYT). These models are therefore reliable especially at low redshifts of $z<1$ (Yoshii \& Takahara 1988) where we constrain the cosmological parameters from the CFRS data. A global rate of star formation is also calculated and is used to discuss an early evolution of galaxies from some recent observations of star formation rate at $z>1$.

\section{Theoretical Model of the Luminosity Density Evolution}

Let $\mathcal{L}(t, \lambda)$ be the comoving luminosity density of galaxies measured at time $t$ from the epoch of galaxy formation and at rest-frame wavelength $\lambda$ per unit wavelength. Then 
$\mathcal{L}(t, \lambda)$ is expressed as, after summing over galaxy types,

$$
\mathcal{L}(t, \lambda)=\sum_{k} \int d L_{B} \psi_{k}\left(L_{B}\right) L_{k}\left(t, \lambda ; L_{B}\right),
$$

where $\psi_{k}\left(L_{B}\right)$ is the present-day luminosity function for blue-selected galaxies of $k$-th type and $L_{k}\left(t, \lambda ; L_{B}\right)$ is the luminosity at $t$ and $\lambda$ per unit wavelength for a galaxy of $k$-th type whose present-day $B$-band luminosity is $L_{B}$. Assuming that the SED evolution $f_{k}(t, \lambda)$ depends only on the galaxy type and not on the luminosity of each galaxy, we write

$$
L_{k}\left(t, \lambda ; L_{B}\right)=\left[\frac{f_{k}(t, \lambda)}{f_{k}\left(T_{G}, \lambda_{B}\right)}\right] L_{k}\left(T_{G}, \lambda_{B} ; L_{B}\right),
$$

where $T_{G}$ is the present age of galaxies and $\lambda_{B}=4400 \AA$. We note that the assumption of luminosity-independent evolution is consistent with the method of evaluating the luminosity density from the CFRS data where only galaxies brighter than $\sim L^{*}$ are sampled at $z \gtrsim 0.5$ and the luminosity function is extrapolated to fainter magnitudes with its shape kept constant (Lilly et al. 1996). Thereby, the luminosity density at $t$ and $\lambda$ can be related to the present-day $B$-band luminosity density of each type:

$$
\mathcal{L}(t, \lambda)=\sum_{k}\left[\frac{f_{k}(t, \lambda)}{f_{k}\left(T_{G}, \lambda_{B}\right)}\right] \mathcal{L}_{k}\left(T_{G}, \lambda_{B}\right) .
$$

We classify galaxies into E/S0, Sab, Sbc, Scd, and Sdm which evolve with different star formation histories. The evolving SEDs of spiral galaxies are given in AYT over a range of wavelength spanning from 1550 to $34000 \AA$, and those for elliptical galaxies are taken from the $10^{12} M_{\odot}$ (baryon mass) model in AY for $3600-34000 \AA$. The ultraviolet SED of elliptical galaxies, the origin of which is still a matter of debate, is prescribed in the same way as in Yoshii \& Peterson (1991), but the following results are hardly changed by this uncertain UV-prescription because E/S0 galaxies contribute little to the total luminosity density at $\lambda<3600 \AA$ as long as redshifts of $z<1$ are concerned in our analysis.

In the galaxy evolution models, stars are assumed to be formed at a rate proportional to a power of mass fraction of gas in a galaxy such as $C(t)=\nu f^{n}(t)$. The rate coefficient $\nu$ is chosen in a way to reproduce the present-day colors of each galaxy type assuming $T_{G}=15$ Gyrs and the power index $n$ is generally believed to lie in the range of 1-2 (see, e.g., Kennicutt 1989). We consider four different evolution models for spiral galaxies such as S1, S2, I1, and I2 (for details see AYT). The symbol 'S' refers to the simple, closed-box model, while 'I' to the infall model which allows for material infall into the disk region. The number attached to ' $\mathrm{S}$ ' and ' $\mathrm{I}$ ' is the adopted value of power index $n$ in $C(t)$. The above four models are considered to cover a range of possible star formation histories and 
are used to assess the uncertainty associated with the prescriptions of galaxy evolution in our analysis.

In order to compare with the CFRS data, we set the normalization as $\mathcal{L}\left(T_{G}, \lambda_{B}\right)=10^{19.296} h_{50} \mathrm{~W} \mathrm{~Hz}^{-1} \mathrm{Mpc}^{-3}$, where $h_{50} \equiv H_{0} /(50 \mathrm{~km} / \mathrm{s} / \mathrm{Mpc})$, following Lilly et al. (1996). The contribution from each morphological type in $\mathcal{L}\left(T_{G}, \lambda_{B}\right)$ is evaluated from the Schechter parameters $\left(\phi_{k}^{*}, L_{B, k}^{*}, \alpha_{k}\right)$ determined by Efstathiou, Ellis, \& Peterson (1988) for the Anglo-Australian Redshift Survey (AARS), the Kirshner-Oemler-Schechter survey (KOS), the Revised Shapley-Ames sample (RSA), and the Center for Astrophysics redshift survey (CfA). The Schechter parameters, converted into the same $B$-band system for the four redshift surveys, are summarized in Table 1 of Yoshii \& Takahara (1989) and are used to calculate the proportion of local luminosity density among galaxy types as $\mathcal{L}_{k}=\phi_{k}^{*} L_{k}^{*} \Gamma\left(\alpha_{k}+2\right)$. The results are tabulated in Table 11. When spiral galaxies are not classified into the four subclasses in the AARS, KOS, and RSA surveys, the relative proportion among the subclasses is assumed to be the same as that for the CfA survey. Fig. 11 shows the luminosity density evolution as a function of time for each galaxy type assuming the CfA mix and $T_{G}=15$ Gyrs. In this figure the S1 models are used for the evolution of spiral galaxies. Unless otherwise stated, the CfA mix is used below as a standard choice.

\section{Probing the Cosmological Parameters}

We consider three representative cosmological models: the Einstein-de Sitter (EdS) universe with $\left(\Omega_{0}, \lambda_{0}\right)=(1,0)$, an open universe with $(0.2,0)$, and a $\Lambda$-dominated, flat universe with $(0.2,0.8)$. The redshift of galaxy formation is assumed to be $z_{F}=5$. Different values of $z_{F}$ hardly change our result as far as we restrict ourselves in $z<1$ (see discussion in $\oint$ 团). The Hubble constant $h \equiv H_{0} /(100 \mathrm{~km} / \mathrm{s} / \mathrm{Mpc})$ is taken as $0.5,0.6$, and 0.7 for the three cosmological models in order to give a reasonable age of galaxies $(12.1,12.4$, and 13.6 Gyrs, respectively). Although the galaxy evolution models reproduce the present-day SED at $T_{G}=15$ Gyrs, we can use these models for the case of $T_{G}=10-15$ Gyrs as well, because the luminosity evolution becomes very weak and changes the SED very little after $t \sim 10$ Gyrs.

In order to compare with the CFRS data obtained for $z<1$, we transform the theoretical predictions into those expressed in terms of $z$. In Fig. 2, we show the comoving luminosity densities at $2800 \AA, 4400 \AA$, and $1 \mu \mathrm{m}$ as a function of $z$ for the EdS universe (left panel), the open universe (middle panel), and the $\Lambda$-dominated universe (right panel). The CFRS data points are those from the "luminosity-function-estimated" values (Lilly et al. 1996) for $2800 \AA$ (circles), $4400 \AA$ (triangles), and $1 \mu \mathrm{m}$ (squares). The four theoretical curves for each waveband correspond to the variation of the evolution models of spiral 
galaxies: S1(solid), S2(dashed), I1(dot-short-dashed), and I2(dot-long-dashed). In this figure all the predicted and observed luminosity densities are shown after scaled to $h=0.5$, and the theoretical curves are made to agree with the $4400 \AA$ data at $z=0$.

The behaviors of theoretical curves are not very different among the three cosmological models because the elapsed time between $z=0$ and 1 is roughly the same as 8 Gyrs. On the other hand, the observed luminosity densities, which have been derived from the direct observables of apparent luminosities and redshifts of galaxies, generally depend on the cosmological parameters. It is clear from Fig. 2 that the observed $\mathcal{L}$-evolution at $z=0-1$ becomes flatter with decreasing $\Omega_{0}$ or increasing $\lambda_{0}$, and eventually falls in agreement with the $\Lambda$-dominated universe. In sharp contrast, however, the observed $\mathcal{L}$-evolution is too steep to agree with the EdS or open universe, and such a discrepancy is much more considerable at $1 \mu \mathrm{m}$. We note that the $\mathcal{L}$-evolution is reliably modeled at $1 \mu \mathrm{m}$, offering an ideal cosmological test when compared to shorter wavelengths. This is because the luminosity density at $1 \mu \mathrm{m}$ is always dominated by ellipticals and is quite insensitive to details of recent star formation history in spiral galaxies, as shown in the lower panel of Fig.1.

For the purpose of quantitative comparison, we have calculated the slope index of the $\mathcal{L}$-evolution, $\alpha \equiv d \log \mathcal{L} / d \log (1+z)$, averaged over the range of $z=0-1$ for each of the galaxy evolution models (S1-I2). We have repeated calculations with type mixes other than the CfA mix in Table 1, but the resulting $\alpha$ is changed at most by 0.08 . Lilly et al. (1996) estimated the observed slope for the EdS cosmology as $\alpha=3.90 \pm 0.75(2800 \AA)$, $2.72 \pm 0.5(4400 \AA)$, and $2.11 \pm 0.5(1 \mu \mathrm{m})$. Using these observational errors which do not depend on $\Omega_{0}$ and $\lambda_{0}$, we have performed a $\chi^{2}$ analysis for $\alpha$ and found that the EdS and open cosmologies are inconsistent with the data with $99.86 \%$ C.L. and 98.6\% C.L., respectively. Assuming a flat universe $\left(\Omega_{0}+\lambda_{0}=1\right)$ with $h=0.6$, the lower limit on $\lambda_{0}$ is obtained as 0.37 (99\% C.L.) or 0.53 (95\% C.L.). Here, one of the galaxy evolution models (S1-I2) is used that gives the most conservative result in the $\chi^{2}$ analysis.

\section{Discussion}

Our conclusion supporting the $\Lambda$-dominated universe is drawn from using the AY and AYT models of galaxy evolution based on fairly standard assumptions on galaxy evolution and local properties of galaxies. However, some other scenarios of galaxy evolution have been proposed so far mainly to solve the so-called "excess" faint blue galaxies around $B \sim 24$. These include a scenario of widespread merging of galaxies (Broadhurst, Ellis, \& Glazebrook 1992) and a scenario which invokes an exotic intermediate-redshift population 
of dwarf galaxies which have ever faded away (Babul \& Rees 1992). It is worth emphasizing that our analysis in this Letter is based on the luminosity density of galaxies but not on their number density. Luminosity density is generally less sensitive to mergers than number density, because increase of number of galaxies at high redshifts is compensated by the corresponding decrease of luminosity of individual galaxies. The exotic dwarf galaxies may significantly contribute in the number density, but it is doubtful that such a faint population occupies a large fraction in the CFRS galaxies at $0.5 \lesssim z \lesssim 1$. In fact, their HST images exhibit the same range of morphological types as seen locally and the CFRS galaxies at $z \sim 0.75$ are broadly similar to the local galaxy population (Schade et al. 1995), indicating that exotic evolutionary processes do not dominate in luminous galaxies.

The redshift of galaxy formation, though still uncertain, has been assumed to be $z_{F}=5$, because different choices give a convergent result below $z \sim 1$ and do not affect the cosmological test presented in this Letter. Recent progress in direct observations of star-forming galaxies at $z \gtrsim 2$ however provides more useful constraint on $z_{F}$. In Fig. 3, assuming the $\Lambda$-dominated universe with $\left(\Omega_{0}, \lambda_{0}\right)=(0.2,0.8)$, we plot the observed star formation rates (SFRs) in the universe which are compiled by Madau et al. (1996), together with the predicted SFRs with $z_{F}=5$ and 7 assuming $h=0.7$ (for details see Totani, Sato, \& Yoshii 1996). The SFRs, observed or predicted, are shown after scaled to $h=0.5$ in this figure. Thick lines show the SFRs from spiral galaxies, and thin lines from all types including elliptical galaxies. Since the absolute SFRs are uncertain by a factor of $2-3$, we normalize the curves to agree with the data at $z<1$. Inspection of this figure clearly indicates that $z_{F}$ is larger than at least $\sim 4$. According to Madau et al. (1996), the observed SFRs beyond $z=1$ shown in Fig. 3 should be considered as lower limits to the real values. However, since their analysis is based on a sample which covers a significant range of magnitude in the galaxy luminosity function, it may be safe to assume that these lower limits are not very different from the real values. If this is the case, the infall models of spiral galaxies with $z_{F} \sim 4-5$ well explain the SFR trend at $z \sim 2-4$, whereas the model of elliptical galaxies overpredicts the SFRs unless they were formed at $z_{F} \gtrsim 5$.

We point out that elliptical galaxies having high SFRs release metals out of the system into the intergalactic medium (IGM) through galactic winds. Miralda-Escude and Rees (1997) argued that the average metal abundance of $\bar{Z} \sim 2 \times 10^{-4}$, which is observed in the Ly $\alpha$ forests seen in quasar spectra, needs one Type II supernova per $5000 M_{\odot}$ baryons in the IGM and these supernova progenitors should have emitted about 20 ionizing photons per each baryon, which are enough to reionize the universe. In our models, an elliptical galaxy with $M_{\text {baryon }}=10^{12} M_{\odot}$ produces about $2.5 \times 10^{10}$ Type II supernovae during the first 0.7 Gyr (Totani, Sato, \& Yoshii 1996) and then releases metal-enriched gas of about 7 $\%$ of $M_{\text {baryon }}$ into the IGM (AY). If the baryon density in the universe is taken as a typical 
value of $\Omega_{b} h^{2}=0.02$ from the Big-Bang nucleosynthesis, the average metal abundance in the universe is given by $\bar{Z} \sim 1.7 \times 10^{-4} h^{2}$. Since this value agrees with the estimate by Miralda-Escude and Rees (1997), we can regard the elliptical galaxies as a source of supplying not only the metals in the IGM but also the ionizing photons which reionize the universe.

We would like to thank an anonymous referee for useful comments. This work has been supported in part by the Grant-in-Aid for the Center-of-Excellence (COE) Research (07CE2002) and for the Scientific Research Fund (05243103, 07640386, 3730) of the Ministry of Education, Science, and Culture in Japan.

\section{REFERENCES}

Arimoto, N. \& Yoshii, Y. 1987, A\&A, 173, 23 (AY)

Arimoto, N., Yoshii, Y., \& Takahara, F. 1992, A\&A, 253, 21 (AYT)

Babul, A. \& Rees, M. J. 1992, MNRAS, 255, 346

Broadhurst, T. J., Ellis, R. S., \& Glazebrook, K. 1992, Nature, 355, 55

Efstathiou, G., Ellis, R. S., \& Peterson, B. A., 1988, MNRAS, 232, 431.

Ellis, R. S., Colless, M., Broadhurst, T., Heyl, J., \& Glazebrook, K. 1996, MNRAS, 280, 235.

Kennicutt, R. C. 1989, ApJ, 344, 685

Lilly, S. J., Tresse, L., Hammer, F., Crampton, D., \& Fèvre, O. Le. 1995, ApJ, 455, 108

Table 1: Local Luminosity Density Proportion in Galaxy Types (4400^)

\begin{tabular}{lccccc}
\hline \hline & \multicolumn{5}{c}{ Relative proportion } \\
\cline { 2 - 6 } Sample & E/S0 & Sab & Sbc & Scd & Sdm \\
\hline AARS... & 0.14 & \multicolumn{4}{c}{0.86} \\
KOS..... & 0.21 & \multicolumn{4}{c}{0.79} \\
RSA.... & 0.20 & 0.54 & \multicolumn{2}{c}{0.26} \\
CfA...... & 0.28 & 0.19 & 0.32 & 0.14 & 0.066 \\
\hline
\end{tabular}


Lilly, S. J., Fèvre, O. Le, Hammer, F., \& Crampton, D. 1996, ApJ, 460, L1

Madau, P., Ferguson, H. C., Dickinson, M. E., Giavalisco, M., Steidel, C. C., \& Fruchter, A. 1996, MNRAS, 283, 1388

Miralda-Escude, J. \& Rees, M. J. 1997, ApJ 478, L57

Schade, D., Lilly, S. J., Crampton, D., Hammer, F., Fèvre, O. Le., \& Tresse, L. 1995, ApJ 451, L1

Tinsley, B. M. 1980, Fund. Cosmic Physics, 5, 287

Totani, T., Sato, K. \& Yoshii, Y. 1996, ApJ, 460, 303

Yoshii, Y. \& Takahara, F. 1988, ApJ, 326, 1.

Yoshii, Y. \& Takahara, F. 1989, ApJ, 346, 28.

Yoshii, Y. \& Peterson, B. A. 1991, ApJ, 372, 8

This preprint was prepared with the AAS IATEX macros v4.0. 


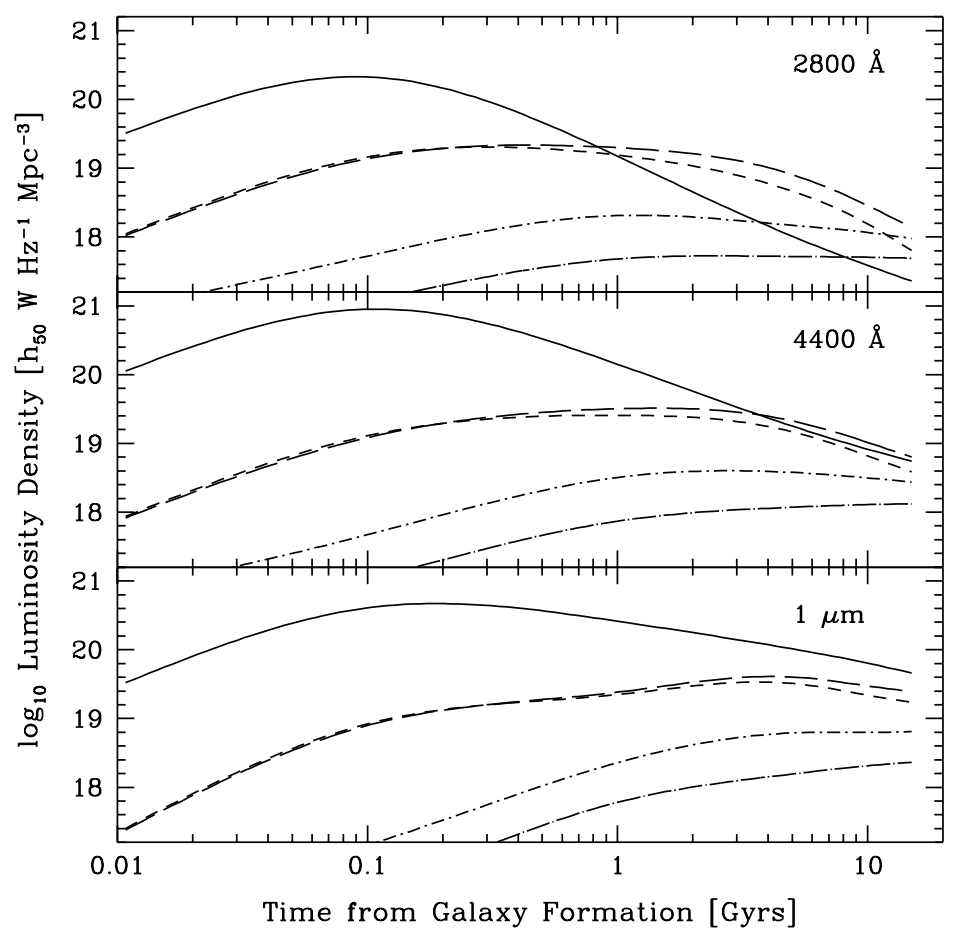

Fig. 1.- Evolution of the comoving luminosity density at $2800 \AA, 4400 \AA$ and $1 \mu \mathrm{m}$ as a function of time for model galaxies of different morphological types. The galactic wind model from AY is used for E/S0 (solid line), and the S1 models from AYT for spiral galaxies of Sab (short-dashed), Sbc (long-dashed), Scd (dot-short-dashed), and Sdm (dotlong-dashed). The luminosity density at 15 Gyrs for the composite of all types is normalized to $\mathcal{L}(4400 \AA)=10^{19.296} h_{50} \mathrm{~W} \mathrm{~Hz}^{-1} \mathrm{Mpc}^{-3}$, and the relative contribution among the types is adopted from the CfA mix in Table 1. 


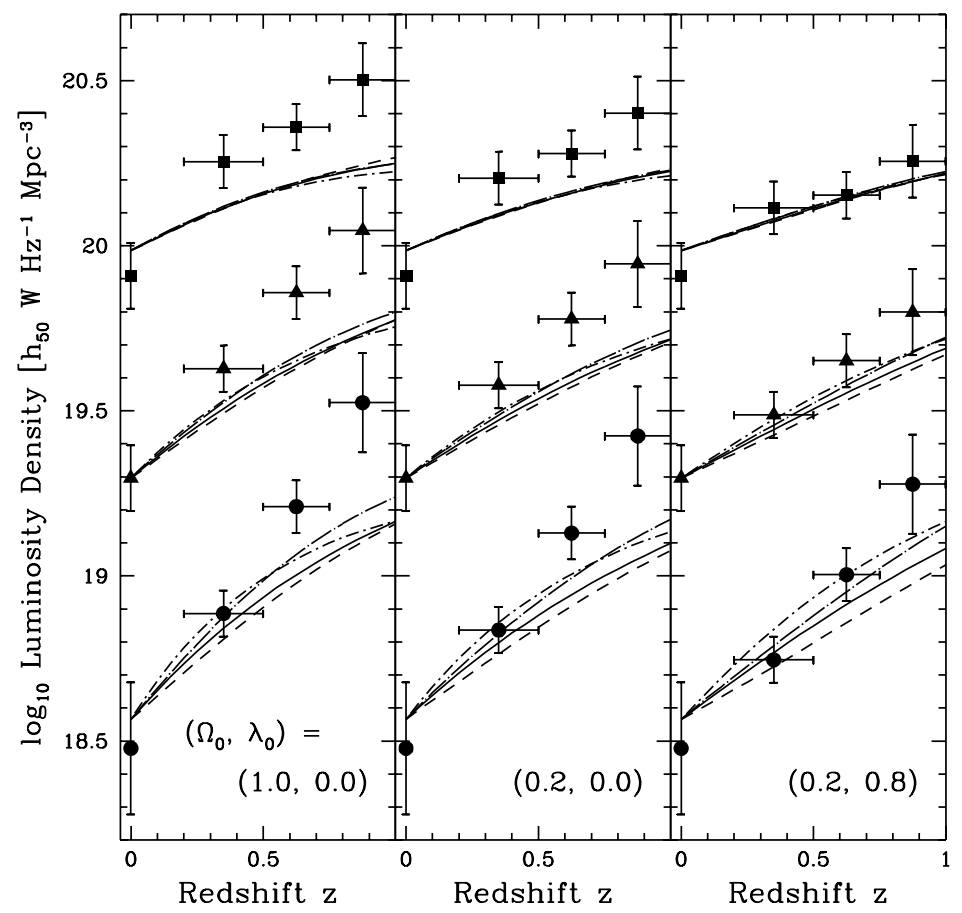

Fig. 2.- Evolution of the comoving luminosity density as a function of redshift for the composite of all galaxy types, shown for the Einstein-de Sitter universe with $\left(\Omega_{0}, \lambda_{0}\right)=(1,0)$ (left panel), the open universe with $(0.2,0)$ (middle panel), and the $\Lambda$-dominated flat universe with $(0.2,0.8)$ (right panel). The CFRS data points (Lilly et al. 1996), scaled to $h=0.5$, are shown by circles $(2800 \AA)$, triangles $(4400 \AA)$, and squares $(1 \mu \mathrm{m})$. The four theoretical curves for each of $2800 \AA, 4400 \AA$, and $1 \mu \mathrm{m}$ correspond to the evolution models of S1 (solid line), S2 (dashed), I1 (dot-short-dashed), and I2 (dot-long-dashed) for spiral galaxies. The galactic wind model for elliptical galaxies is used in common. The curves are normalized to coincide with the $4400 \AA$ data at $z=0$. 


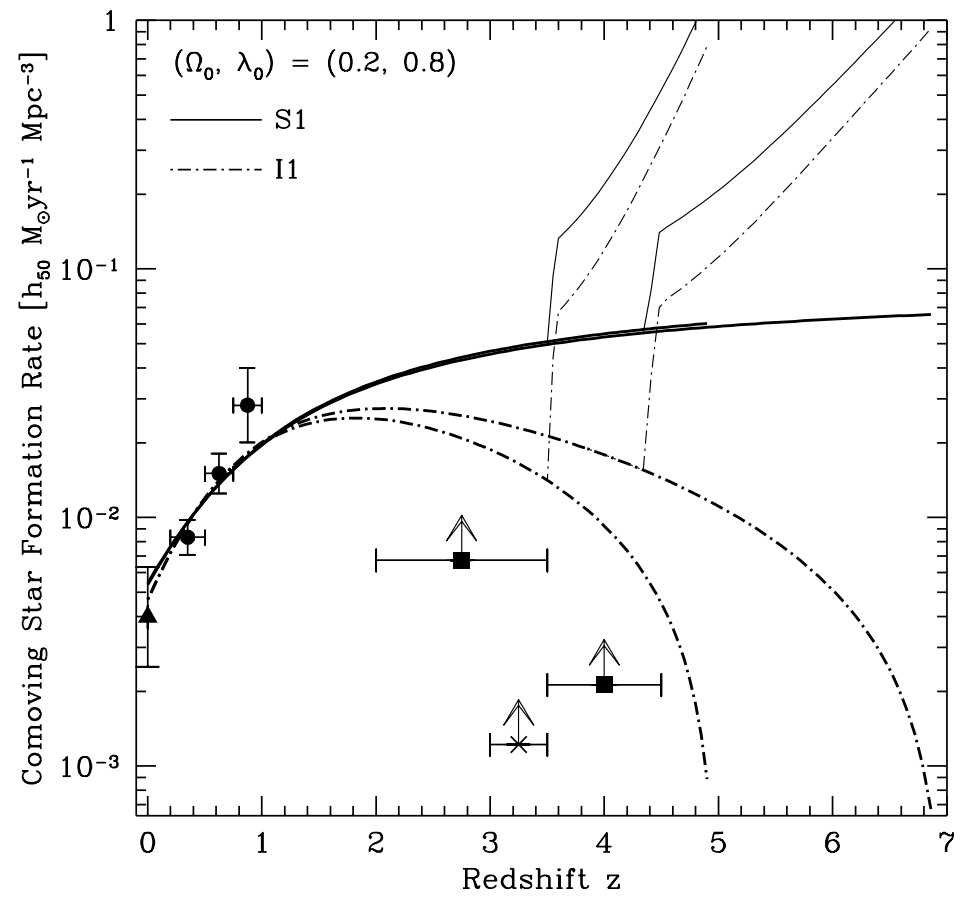

Fig. 3.- Star formation rate (SFR) in the universe as a function of redshift. The $\Lambda$ dominated flat universe with $\left(\Omega_{0}, \lambda_{0}\right)=(0.2,0.8)$ is assumed for both predicted and observed SFRs shown in this figure after scaled to $h=0.5$. The data points are those compiled by Madau et al. (1996). The theoretical curves are based on the S1 (solid) or I1 (dot-dashed) models for spiral galaxies and the galactic wind model for elliptical galaxies. Galaxies of all types are assumed to be formed at $z_{F}=5$ or 7 , with $h=0.7$. The SFRs from spiral galaxies only are shown by thick lines, while those from all types including elliptical galaxies by thin lines. The theoretical curves are normalized to agree with the data at $z<1$. 